\title{
Movement of Arginine Through OprD: The Energetics of Permeation and The Role of Lipopolysaccharide in Directing Arginine to The Protein
}

Firdaus Samsudin ${ }^{1}$ and Syma Khalid ${ }^{1 *}$

${ }^{1}$ School of Chemistry, University of Southampton, Southampton SO17 1BJ, UK

*Corresponding author: s.khalid@soton.ac.uk

\begin{abstract}
The outer membrane channel OprD from Pseudomonas aeruginosa transports basic amino acids and clinically relevant carbapenem antibiotics. Understanding the molecular basis of substrate permeation across this channel will therefore lead to better therapeutic designs to treat infections. Using umbrella sampling simulations, we calculated the potential of mean force (PMF) for the arginine permeation pathway through OprD. The PMF reveals a deep free energy well of $\sim 6 k T$ around the putative substrate binding site followed by a shallower well of $\sim 4 k T$ close to the most constricted region of the pore. Despite becoming partially dehydrated during translocation, some water molecules are retained to shield the guanidinium side chain of arginine from the ladder of basic residues in the protein. Sugars of the lipopolysaccharide headgroups form contacts with arginine and could potentially play an important role in transferring substrate from the external medium to OprD. The PMF through bulk membrane shows a large energetic barrier of $\sim 45 k T$ within the hydrophobic core of the membrane, suggesting that spontaneous translocation without OprD is highly unlikely. This significant energetic penalty is likely caused by the extensive distortion of the lower leaflet of the outer membrane as phospholipid headgroups sink inwards to interact with charged groups of arginine. Our results provide quantitative insights into solute permeation across bacterial outer membrane.
\end{abstract}




\section{Introduction}

The outer membrane of Gram-negative bacteria acts as a strong shield against attack by noxious external compounds such as antibiotics. The thick layer of sugar moieties on lipopolysaccharides (LPS) impedes diffusion of lipophilic molecules, whilst the hydrophobic membrane core is impermeable to polar solutes. Over the years, the delivery of drugs across the outer membrane exploits the presence of numerous outer membrane porins-non-specific water-filled channel proteins-that exist to allow uptake of nutrients and extrusion of waste products. ${ }^{1,2}$ Several penicillins and cephalosporins, for example, have been shown to be translocated by OmpF in Escherichia coli. ${ }^{3}$ Understanding the molecular details of solute permeation via outer membrane porins is therefore beneficial for development of novel antibacterial agents.

Pseudomonas aeruginosa is a major pathogenic Gram-negative bacterium that is one of the leading causes of nosocomial infections and is prevalently associated with lung infections amongst cystic fibrosis patients. ${ }^{4}$ It shows remarkably high intrinsic resistance to common antibiotics largely due to the low permeability of its outer membrane. 5,6 The reduced influx of antibiotics then allows for more efficient secondary resistance mechanisms including drug efflux pumps and $\beta$-lactamase. Unlike Enterobacteria, $P$. aeruginosa does not express general porins like OmpF, but instead utilises various substrate specific channels for nutrient uptake. ${ }^{7}$ While these channels have narrower pores compared to porins, substrate binding occurs at higher affinities due to their distinct binding sites, which is advantageous in nutrition-poor environments where these bacteria are found. The majority of small molecules are imported via proteins from the outer membrane carboxylate channels (Occ) family, ${ }^{8}$ which is further divided into two subfamilies: OccD and OccK. The former transports basic amino acids and the latter prefer cyclic compounds. The prototypical member of OccD subfamily, OprD (also known as OccD1), is of great pharmaceutical interest as in addition to mediating the uptake of basic amino acids, it also allows passage of carbapenem $\beta$-lactam antibiotics. ${ }^{9,10}$ Recently, whole cell based assays revealed that even minor modifications to the chemical structure of novel carbapenem analogues can have significant effects on OprD-mediated uptake, ${ }^{11}$ further indicating the importance of understanding the molecular mechanism underlying substrate recognition and permeation.

The structure of $P$. aeruginosa OprD has been solved by X-ray crystallography. $8,12,13$ OprD is a monomeric 18-stranded $\beta$-barrel protein with several 
large external loops, two of which; L3 and L7, fold into the barrel to form a narrow constriction. A line of positive charges formed by five arginine and one lysine residues (also known as a "basic ladder") is found on one side of the pore, while the other side is predominantly negatively charged, creating an asymmetric charge distribution along the substrate permeation pathway. The basic ladder is a characteristic of all Occ channels, ${ }^{8,14}$ and is also found in the phosphate-specific channel OprP. ${ }^{15}$ The crystal structure of OpdF (OccK2) bound to its substrate, glucuronate, reveals salt bridges between the substrate carboxyl moiety and two of the arginine residues, suggesting that the basic ladder acts as an electrostatic conduit that guides substrates permeation via interactions to their carboxyl group. ${ }^{13}$ Mutation of either one of three of these basic residues (R389, R391 and R410) in OprD significantly decreases substrate influx. ${ }^{13}$ Steered molecular dynamics (MD) simulations suggest that arginine adopts a specific orientation along the translocation pathway that maximises interactions between its carboxylate and these basic residues. ${ }^{16}$ As the crystal structure of OprD bound to a basic amino acid has not yet been determined, it is not known how the positively charged side chain of a substrate is accommodated during permeation. Superposition of OprD with OpdF-glucuronate structure shows that the equivalent residues in OprD to which the sugar rings of glucuronate bound in OpdF form a negatively charged pocket. These "eyelet" residues, Y176, Y282 and D307, are found opposite to the basic ladder. Triple mutation to make the pocket positively charged substantially reduced uptake activity of arginine and change OprD into a glutamate selective channel, ${ }^{13}$ demonstrating its importance for substrate recognition. Furthermore, docking studies followed by MD simulations showed arginine binds to a well-defined region nearby these eyelet residues. ${ }^{13}$

These structural, mutational and computational studies have provided a qualitative picture of substrate recognition in OprD; however, a robust quantitative analysis on the energetics of substrate permeation across this channel is still missing. Delineating regions of energetically favourable binding would likely be beneficial for rational drug design targeting this channel. Calculation of potential of mean force (PMF) using enhanced sampling methods such as umbrella sampling ${ }^{17-19}$ and adaptive force biasing ${ }^{20,21}$ is the state-of-the-art method to address this question. While these methods have been used to characterise the free energy of transport of ions and small solutes, basic amino acids (for which OprD is selective), pose a bigger challenge due to their larger size. Here we employed umbrella sampling with a total of 
$\sim 36 \mu$ s of atomistic simulations to calculate the PMF along the translocation pathway of arginine via either OprD or bulk outer membrane. Additionally, our simulations uncover important molecular interactions experienced by arginine, not only with OprD, but also with surrounding lipid and water molecules as it attempts to cross the outer membrane.

\section{Methods}

\section{Systems construction}

The crystal structure of OprD was obtained from the Protein Data Bank (PDB: 3SY7) ${ }^{8}$ and missing residues were added as described in a previous study. ${ }^{13}$ The structures of OprD R410A and Y176R/Y282R/D307H mutants were generated using PyMOL. ${ }^{22}$ The arginine ladder was protonated as would be expected at physiological $\mathrm{pH}$, in a polar environment (the protein interior). The outer membrane model was based on that of E. coli K12 strain containing Ra LPS lipids in the upper leaflet ${ }^{23,24}$ and 1palmitoyl 2-cis-vaccenic phosphatidylethanolamine (PVPE), 1-palmitoyl 2-cisvaccenic phosphatidylglycerol (PVPG) and 1-palmitoyl 2-cis-vaccenic 3-palmitoyl 4cis-vaccenic diphosphatidylglycerol (PVPV; also known as cardiolipin) in a 18:1:1 ratio in the lower leaflet. ${ }^{25-28}$ This model was developed by Piggot et al. ${ }^{29}$ and has been extensively used for atomistic simulations of outer membrane proteins. ${ }^{30-32}$ The OprD structure was embedded into the outer membrane model using the g_membed protocol. ${ }^{33}$ The protein-membrane complex was solvated, $\mathrm{Mg}^{2+}$ ions were added to aid cross-linking between the LPS head groups and $\mathrm{Cl}^{-}$ions were used to neutralize the system as in our previous work. ${ }^{29}$ For simulations of arginine permeation, an arginine molecule was positioned in bulk solution approximately $3 \mathrm{~nm}$ above OprD. Each system underwent a short 1 ns equilibration simulation whereby positional restraints were imposed on all atoms of the protein and arginine substrate. These restraints were removed for subsequent simulations.

\section{Equilibrium MD simulations}

Two repeats of 500 ns simulation were performed on the apo system (without arginine substrate) starting with different velocity assignment. Temperature was maintained at $310 \mathrm{~K}$ using the velocity rescale thermostat with a time constant of $0.1 \mathrm{ps}$. ${ }^{34}$ Pressure was kept at $1 \mathrm{~atm}$ using a semi-isotropic coupling to a Parrinello-Rahman barostat using a time constant of 2 ps. ${ }^{35}$ To allow for a 2 fs integration time step, all bonds were 
constrained using the LINCS algorithm. ${ }^{36}$ The short range electrostatic and van der Waals cut-offs were set to $1.4 \mathrm{~nm}$, whilst the long range electrostatic interactions were approximated using the particle mesh Ewald method. ${ }^{37}$ All simulations were performed using the GROMACS 5 code, ${ }^{38}$ GROMOS 54 A7 force field ${ }^{39}$ and SPC water model. ${ }^{40}$ Visualisation was performed with Visual Molecular Dynamics. ${ }^{41}$

\section{Steered and umbrella sampling MD simulations}

A harmonic spring with a force constant of $25 \mathrm{~kJ} \mathrm{~mol}^{-1} \mathrm{~nm}^{-2}$ was attached to the centre of mass of the arginine molecule and this was pulled along the z-axis (perpendicular to the plane of the membrane) at a constant velocity of $0.5 \mathrm{~nm} \mathrm{~ns}^{-1}$. No restrained was applied to the arginine molecule in the $x$-and $y$-axis during the pulling simulations. This $20 \mathrm{~ns}$ pulling simulations generated around 2000 snapshots, which are distributed evenly along the axis of the channel. Umbrella sampling windows were then selected along the reaction coordinate (from 5 to $-4 \mathrm{~nm}$ along the $z$ axis) with a separation of $0.1 \mathrm{~nm}$ between windows. For each window, a $200 \mathrm{~ns}$ simulation was performed with the centre of mass of arginine restrained in the vector along the reaction coordinate using a harmonic force constant of $1000 \mathrm{~kJ} \mathrm{~mol}^{-1} \mathrm{~nm}^{-2}$. Extra umbrella sampling windows were added based on the histogram overlap to ensure adequate sampling. A total of 80 and 100 umbrella sampling simulations were performed for arginine permeation across OprD and the outer membrane, respectively. The weighted histogram analysis methods (WHAM) ${ }^{42}$ implemented in GROMACS (gmx wham) ${ }^{43}$ was used to estimate autocorrelation time for each umbrella window and calculate the error on each PMF profile using the Bayesian bootstrapping technique. To probe potential guanidinium pairing between the permeating arginine and the residues of the basic ladder, we reran 14 umbrella sampling windows using GROMOS 54A8 forcefield.

\section{Results \& Discussion}

OprD is Stable inside the Outer Membrane Model. Previous simulation studies of OprD used simplified membrane models help interpret in vitro experimental studies, but are not representative of the Gram-negative bacterial complex outer membrane. ${ }^{13,16,44-46}$ In recent years, compelling experimental evidence has shown that certain components of the outer membrane, particularly LPS and cardiolipins, play a key role in the functioning of outer membrane proteins. ${ }^{47,48}$ In the present study OprD 
was inserted into a model of $E$. coli outer membrane containing LPS in the outer leaflet and a mixture of phospholipids in the inner leaflet. ${ }^{29}$ Two 500 ns equilibrium simulations were performed to ensure the stability of $\mathrm{OprD}$ within this membrane (Figure S1). All-atom root-mean-square deviation (RMSD) of the $\beta$-barrel and the external loops reached a plateau of $\sim 0.3 \mathrm{~nm}$ after $\sim 100 \mathrm{~ns}$, suggesting a stable system. Root-mean-square fluctuation (RMSF) and secondary structure analysis indicate relatively lower flexibility of the external loops compared to previous studies potentially due to the slower dynamics of the surrounding LPS molecules. In particular, Samanta et al. showed that loop L2 acts as a flap that mediates channel opening and closing. ${ }^{46}$ Our simulations, however, displayed no large conformational changes of L2 as it formed hydrogen bonds with the sugar and phosphate groups of nearby LPS molecules. One important caveat in our study is the use of E. coli outer membrane model instead of that from $P$. aeruginosa. Given the largely similar physicochemical properties of LPS molecules in both bacteria, however, it is likely that the interactions between OprD and the LPS headgroups observed in our simulations remained in a $P$. aeruginosa outer membrane. We then used the MOLE software ${ }^{49}$ to determine OprD pore profile (Figure S2). The most constricted region of the pore was $\sim 0.6 \mathrm{~nm}$ wide and located approximately midway along the channel. The side chains of R391 (which is a part of the basic ladder), S130, D295 and S296 flanked this region, and the distance between them were maintained between $0.4-0.8 \mathrm{~nm}$, indicating that the pore remained opened throughout the simulations. This is confirmed by flux analysis, which shows a steady water flow through the channel. 

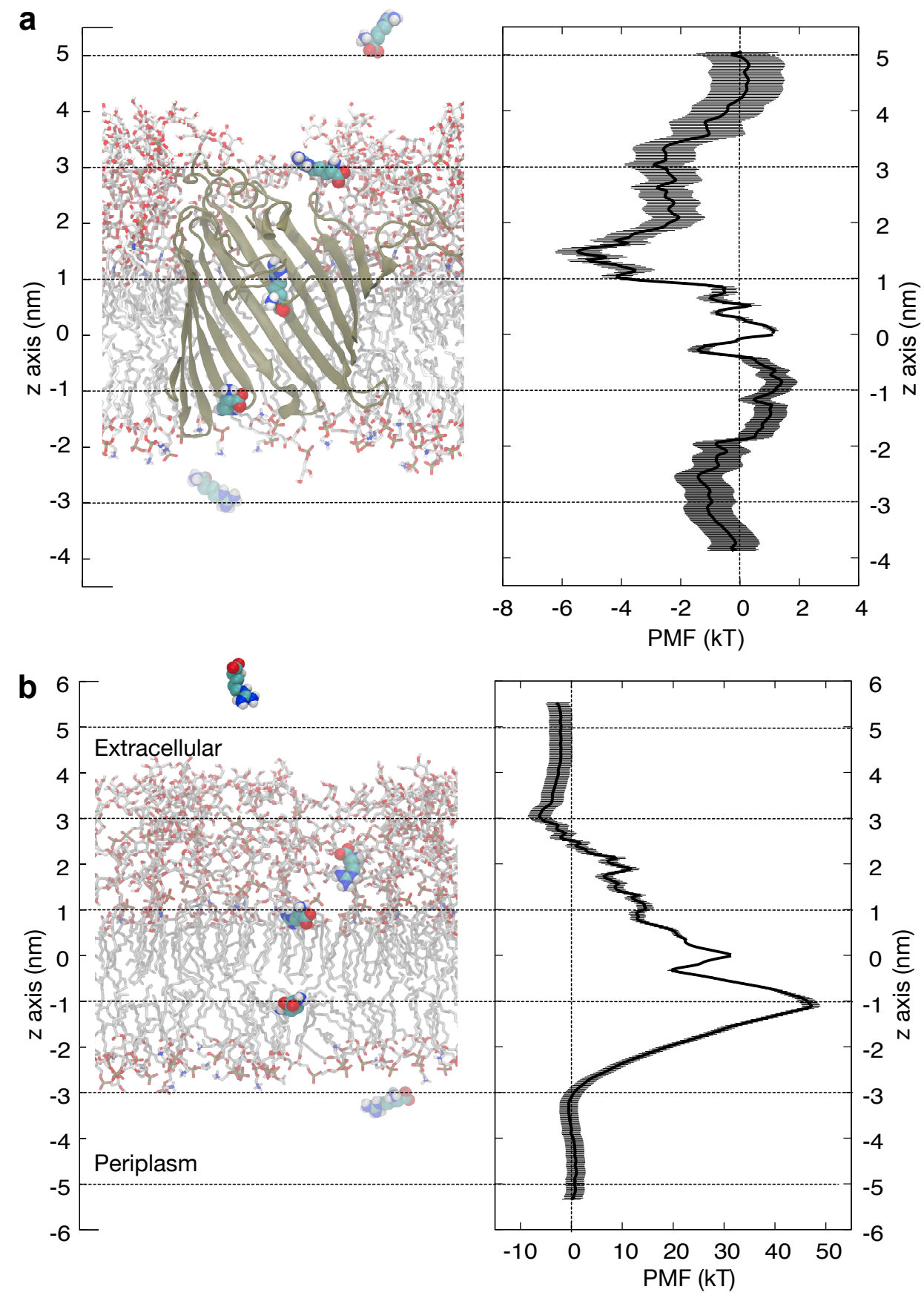

Figure 1. PMF of arginine permeation through $a, O p r D$ and $b$, outer membrane. The grey bars indicate standard deviation estimated from bootstrapping.

Energetics of Arginine Permeation through OprD. Having confirmed that OprD is stable in the outer membrane model, we then performed steered MD simulations to generate a series of configurations along arginine permeation pathway. In these simulations we have only sampled a single protonation state of arginine; however, given its high pKa value arginine molecule is invariably protonated under physiological $\mathrm{pH}$ and therefore this is the state we have used ${ }^{50}$. Similar to the observations reported 
by Parkin et al., ${ }^{16}$ arginine translocation along the pore proceeded in a distinct fashion: first it bound to the negatively charged pocket made by the eyelet residues, and then it aligned almost parallel to the membrane normal as it passed through the most constricted region of the pore with its carboxylate group always pointing toward the basic ladder (Figure S3). We then performed umbrella sampling simulations with the sampling windows separated by $0.1 \mathrm{~nm}$ and each run for $200 \mathrm{~ns}$. To obtain an accurate PMF profile two important criteria were evaluated: sampling and convergence (Figure S4). For the former, we inspected the free energy histogram overlap and found excellent overlaps (more than $10 \%$ between adjacent windows) throughout the entire reaction coordinate, suggesting that adequate sampling has been achieved. To check for convergence PMF profiles were computed using non-intersecting time blocks of the simulations. Convergence was achieved after $100 \mathrm{~ns}$; therefore, the first $100 \mathrm{~ns}$ of the simulations was regarded as equilibration and excluded from subsequent analyses.

The PMF profile reveals no significant energetic barrier along the permeation pathway, but rather features a few wells of different depths (Figure 1a). Cluster analysis was performed to determine the most frequently sampled conformation of arginine from umbrella sampling windows corresponding to these energy wells. The first shallow well of $\sim 3 \mathrm{kT}$ shows interactions between arginine and LPS as well as loop L8 of OprD. Arginine formed hydrogen bonds via its guanidinium, carboxyl and amino groups with the sugar moieties of LPS (Figure 2a and S5). Additionally, the guanidinium group formed ionic interaction with the carboxyl side chain of residue D351 on L8. Further inspection shows that arginine was consistently hydrogen bonded to various parts of the LPS sugar headgroup, and formed intermittent contacts with several polar and charged residues on the external loops of OprD. It is therefore likely that LPS plays a role in capturing arginine from the external medium and promotes initial interactions with OprD. 

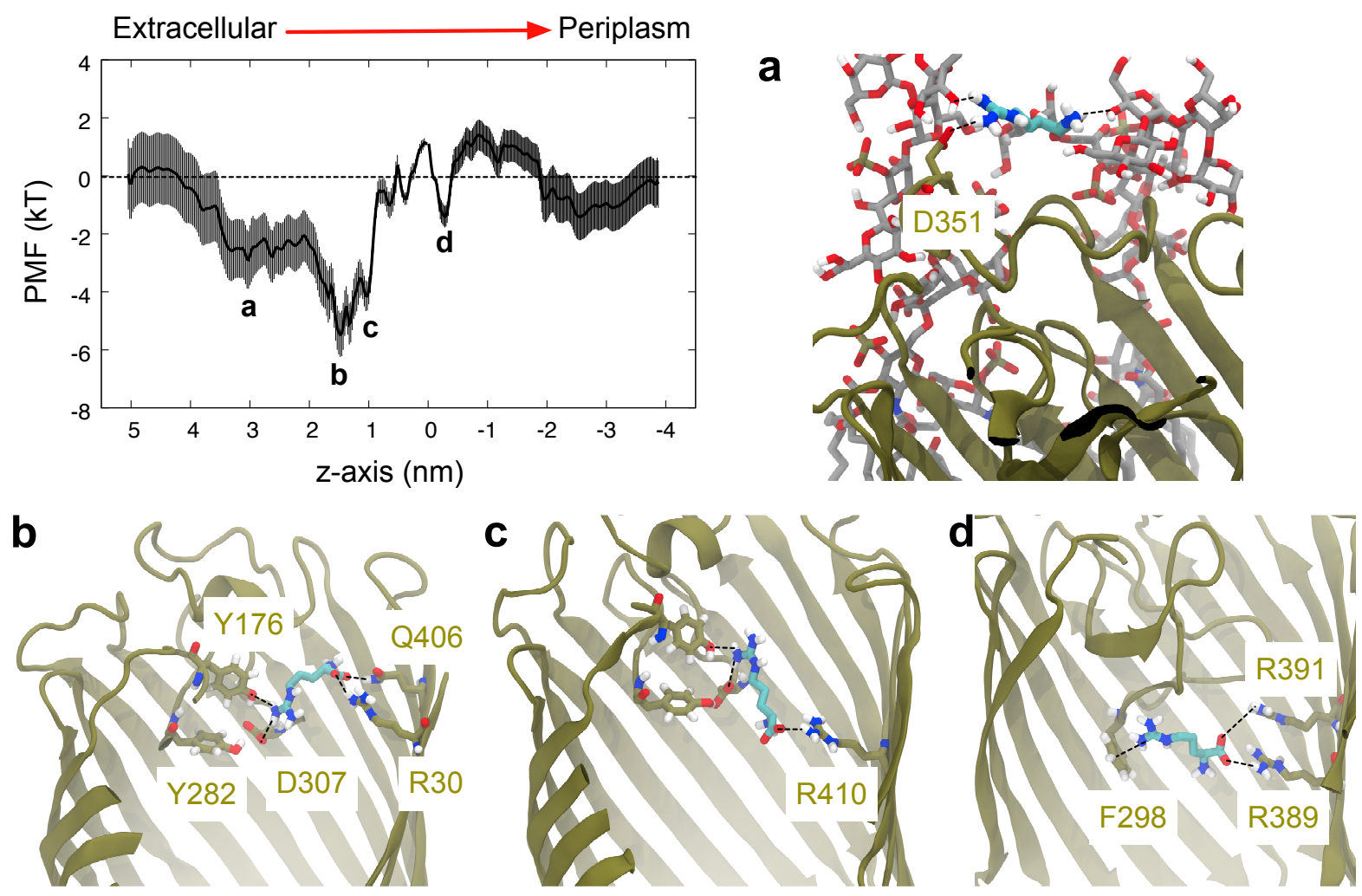

Figure 2. Interactions between arginine and LPS and OprD during permeation. a, b, c and d, Representative snapshots from umbrella sampling windows corresponding to the energy wells labelled on the PMF profile. The red arrow indicates the direction of pulling.

The next energy well is the deepest at $\sim 6 k T$. Unsurprisingly, this corresponds to arginine bound to OprD putative binding site, whereby the guanidinium group inserted into the negatively charged pocket made of the eyelet residues, and the carboxyl group formed a salt bridge with R30, which is a part of the basic ladder (Figure $2 b)$. Also, an adjacent residue Q406 formed a hydrogen bond with the carboxyl moiety. This is consistent with a previous metadynamics study, which shows the presence of an energy minimum for arginine passage in this region. ${ }^{46}$ Intriguingly, arginine could also flip its orientation such that the primary amino group interacted with the eyelet residues (Figure 3). This conformation, however, was less stable as the positively charged guanidinium moiety was in close proximity to the basic ladder. We did not observe any guanidinium-guanidinium pairing interactions, such as those reported by Allolio et al. ${ }^{51}$ This was reproduced by running 14 umbrella sampling windows (the ones in which the arginine is in contact with the residues of the basic ladder) with the Gromos54A8 force-field, ${ }^{52}$ which is reportedly more accurate for charged residues. We reversed the charge of this binding site by mutating the eyelet residues, as 
described in Eren et al., ${ }^{13}$ and performed additional steered MD simulations to understand the implication on arginine permeation. Not only did arginine not make any contact with the now positively charged binding site, it also evaded the basic ladder and translocated through a previously undescribed channel between loop L3 and the barrel. We note that this translocation pathway is likely an artefact of the pulling force. That notwithstanding, these steered MD simulations confirmed the importance of the acidic binding pocket for the correct orientation of arginine vis-à-vis the basic ladder, and subsequently for its permeation through OprD. It is worth noting that this energy well of $\sim 6 k T$ around the arginine binding site is relatively shallow, for example compared to values determined from PMFs of phosphate translocation across OprP, ${ }^{17,20}$ suggesting weaker substrate binding by OprD. This may explain why the co-crystal structure of OprD and its substrate is still elusive.
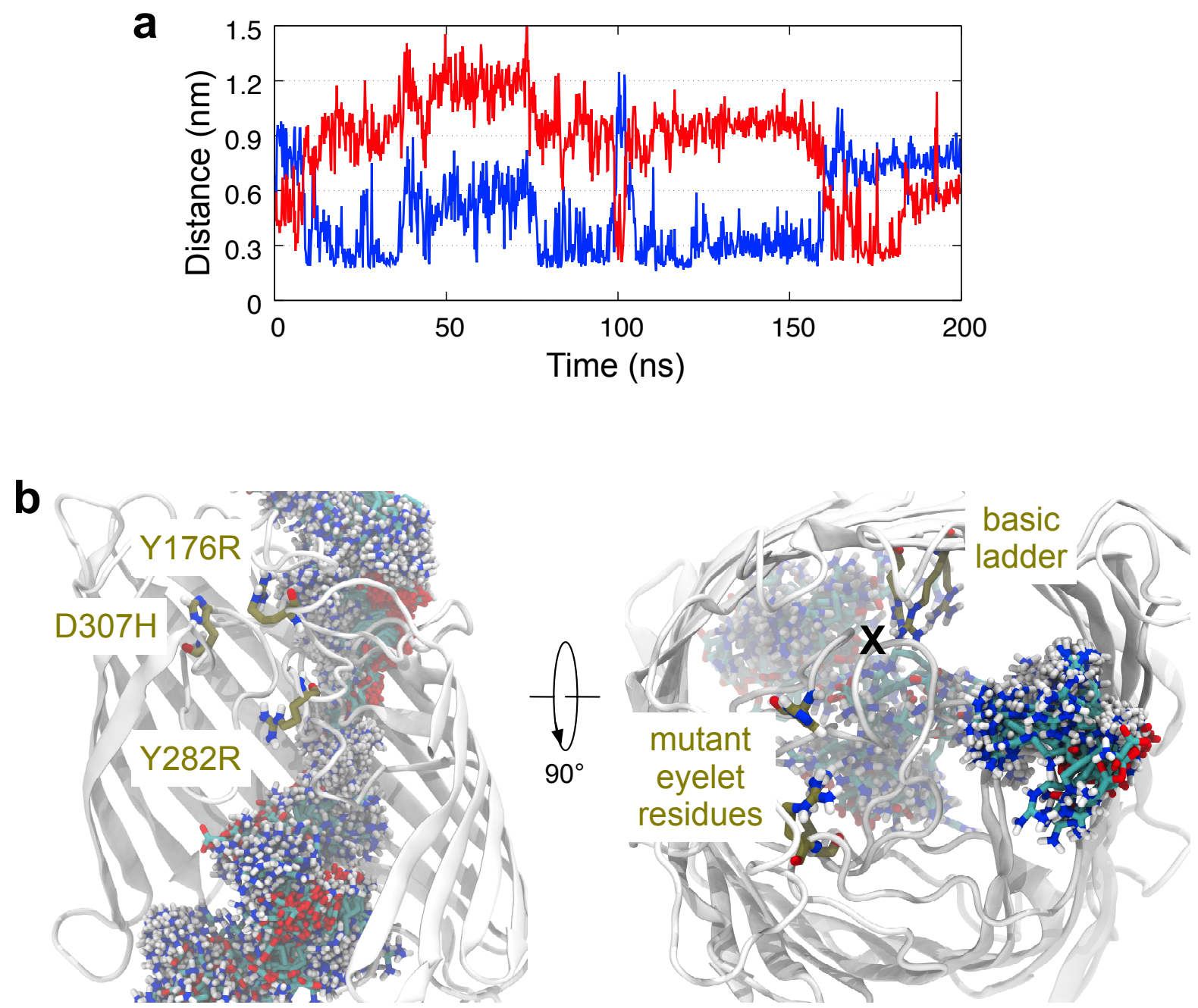

Figure 3. Interactions of arginine with the eyelet residues a, Minimum distance between the guanidinium (blue) and primary amino (red) groups of arginine substrate and the side chains of the eyelet residues throughout a window of the umbrella sampling simulations. b, Side (left) and top (right) 
views of a steered MD simulation performed on Y176R/Y282R/D307H OprD mutant. The translocation pathway for wild-type OprD is indicated by " $X$ ". Snapshots of arginine substrate taken every 100 ps show a significantly different permeation pathway to wild type.

Another energy well of $\sim 4 k T$ was observed prior to arginine passage through the smallest constriction of the channel. In this window, arginine adopted a vertical orientation along the long axis of the channel, whereby the guanidium group maintained its interactions with the previously discussed binding pocket while the carboxyl group slid down the basic ladder to interact with R410 (Figure 2c and 4). This residue is found above R391, which formed the narrowest part of the pore (Figure S2). Arginine retained this upright orientation for $\sim 1 \mathrm{~nm}$ along the translocation pathway as it moved down the basic ladder. We mutated residue R410 to alanine and performed steered MD simulations. Interestingly, this single mutation with the rest of the basic ladder present was enough to disrupt the distinct orientation of arginine during permeation. This distinct upright orientation is crucial to maintain simultaneous interactions of arginine with both the binding pocket and the basic ladder, which is potentially necessary for permeation. This result is in agreement with a previous experimental study that shows mutation of each of R389, R391 and R410 is detrimental to arginine uptake. ${ }^{13}$ After arginine passed through the narrowest constriction of the pore, we observed another shallow well of $\sim 2 k \mathrm{k}$. This corresponds to the carboxyl group interacting with the last residue on the basic ladder, R389 (Figure 2d). The guanidinium moiety was stabilised by residues on loop L7 primarily F298 via a potentially pi-cation interaction. Subsequent windows reveal no further persistent interaction between arginine and $\mathrm{OprD}$, which explains the near zero PMF values, likely due to the wide nature of the channel on the periplasmic side. 

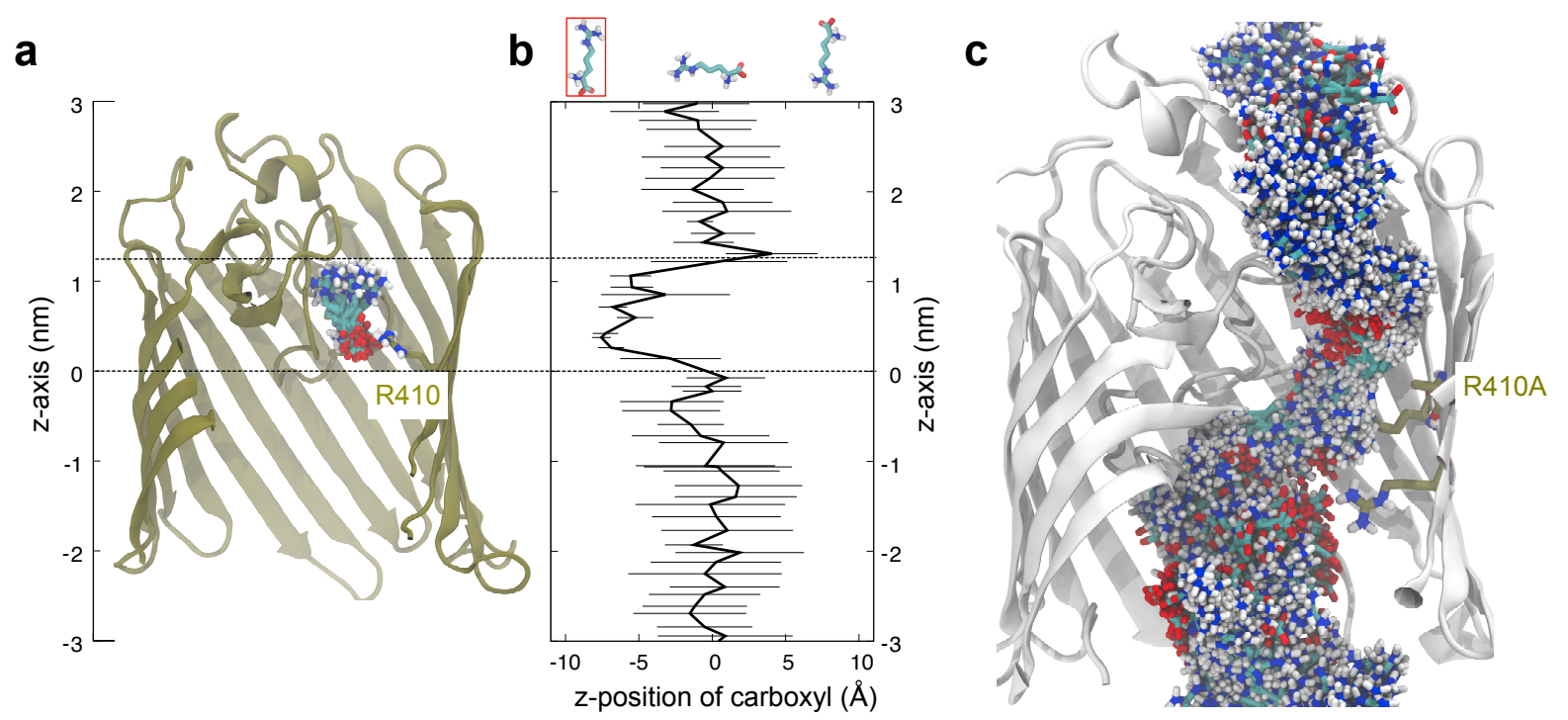

Figure 4. Interactions of arginine with the basic ladder. a, Snapshots of arginine substrate taken every $10 \mathrm{~ns}$ for the last $100 \mathrm{~ns}$ from an umbrella sampling window showing its upright orientation and interaction with R410. $\mathbf{b}$, The orientation of the arginine substrate in all umbrella sampling windows defined by the z-position of the carboxyl group. Error bars indicate standard deviations. Representative structures of these different orientations are shown on top of the graph with the upright orientation depicted in a red box. c, Steered MD simulation was performed on R410A OprD mutant. Snapshots or arginine substrate taken every 100 ps show the loss of its upright orientation during permeation.

Energetics of Arginine Permeation through Outer Membrane. To compare the PMF profile of arginine passage through OprD to that via bulk outer membrane, a similar steered MD and umbrella sampling protocol was performed. As observed in PMF of polar solutes across the outer membrane reported by Carpenter et al., ${ }^{19}$ the PMF of arginine is also asymmetric (Figure 1b). A small well was present in the LPS side whilst large energetic barriers were observed near the hydrocarbon lipid tails. The shallow well of $\sim 4 k T$ corresponds to arginine contacts with the LPS headgroup. We observed several hydrogen bonds formed between the polar groups of arginine and the sugar moieties of LPS, making this configuration energetically favourable (Figure $5 a)$. This is similar to our simulations with OprD, whereby arginine made favourable interactions with the LPS headgroup prior to contacting the OprD external loops (Figure 2a).

As arginine navigated deeper into the membrane and closer towards the hydrophobic core, the PMF increases considerably. Two large peaks were observed, one at $\sim 30 \mathrm{kT}$ and another at $\sim 45 \mathrm{kT}$, for windows corresponding to arginine within the hydrocarbon tails of LPS and phospholipids respectively. Further inspection reveals 
that in the latter, the lower leaflet of the membrane significantly deformed around the arginine molecule, which explains the larger energetic barrier. The phosphate groups of nearby phospholipids moved inward towards arginine resulting in their lipid tails splayed apart (Figure 5b). In the windows where arginine was within the LPS tails no significant deformation was observe, hence the smaller energetic barrier. This is potentially due to the LPS headgroups strongly cross-linked by divalent cations, which prevents their phosphate moieties from sinking into the membrane core.
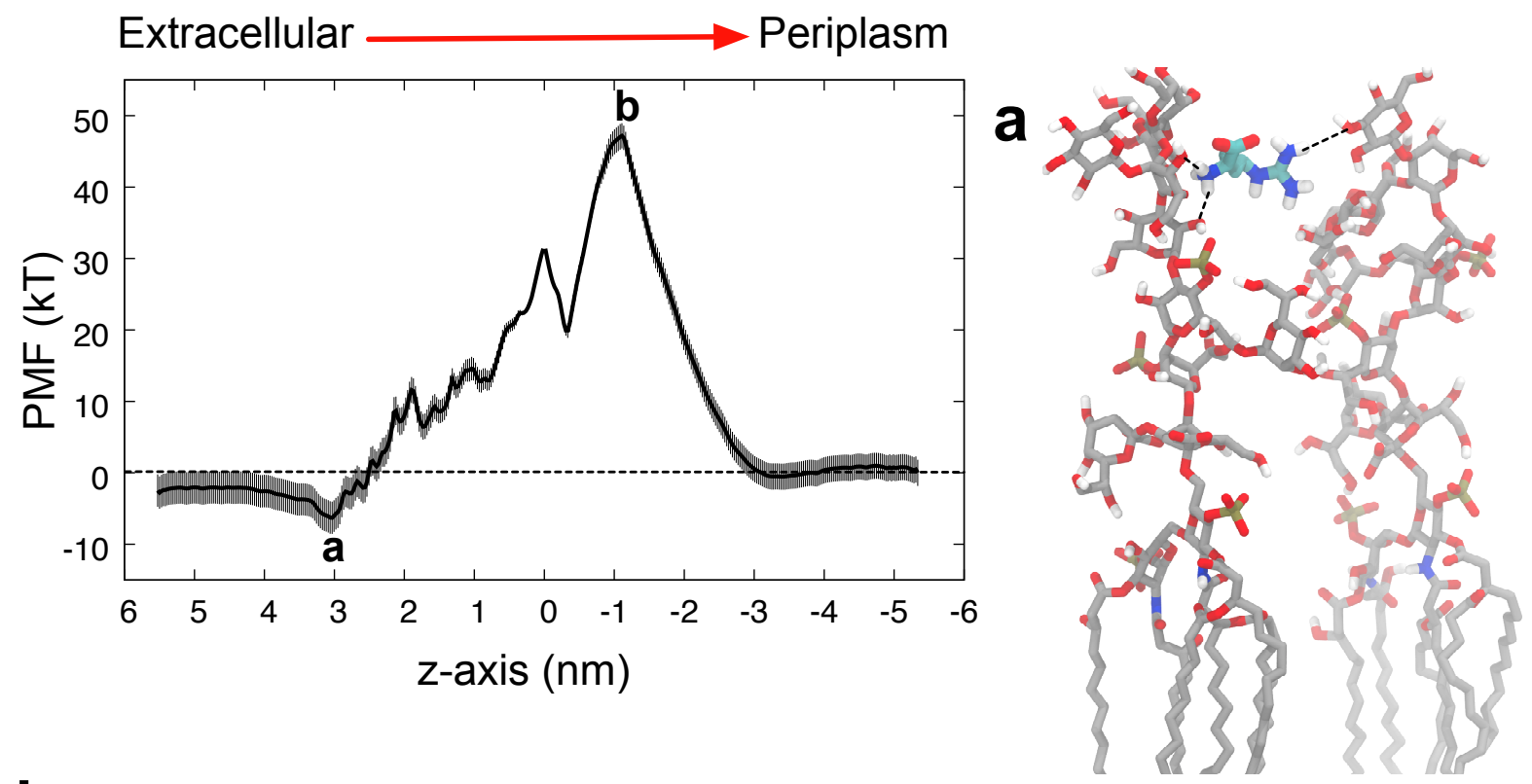

b

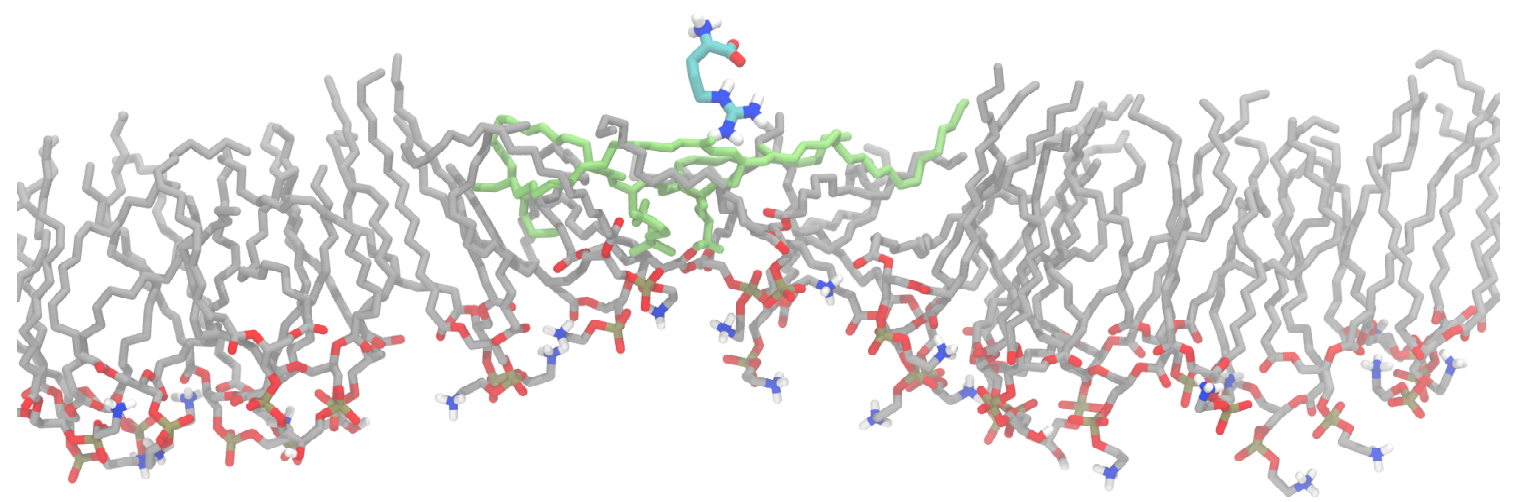

Figure 5. Interactions of arginine with bulk outer membrane. $\mathbf{a}$ and $\mathbf{b}$, Snapshots from umbrella sampling windows corresponding to the labelled energetic well and barrier in the PMF profile. The two closest phospholipids to the arginine molecule in $\mathbf{b}$ are coloured lime green. The red arrow indicates the direction of pulling.

LPS molecules diffuse an order of magnitude slower than phospholipids due to their larger size and cross-linking between their headgroups; ${ }^{29}$ to understand how the different dynamics of the upper and lower leaflets of the outer membrane impact the diffusion of arginine, we calculated the correlation of motion between arginine and 
surrounding lipids in all windows (Figure S6). Interestingly, there was a high correlation between the direction of motion of arginine and surrounding LPS molecules when it permeated the upper leaflet. A similar characteristic was observed in the diffusion of outer membrane proteins. ${ }^{53}$ We tracked the centre of mass motion of arginine in the simulation windows corresponding to LPS headgroups and found that the arginine molecule was trapped between headgroups of four adjacent LPS molecules and was not able to move away and interact with other LPS molecules in the time scale of our simulations. As a result, the arginine motion was strongly coupled to that of LPS. In contrast, in the lower leaflet arginine was able to move between and interact with different phospholipid molecules throughout the simulation due to their faster dynamics and the lack of crosslinks between their headgroups. As such, the direction of motion of arginine in this leaflet shows lower correlation to that of the phospholipids.

The role of water during permeation. Hydration shell often plays an important role during solute permeation across channels and transporters. To understand the role of water during arginine translocation, we calculated the number of water molecules found within $3 \mathrm{~A}$ of arginine in all of the umbrella sampling windows (Figure 6). As many as 18 water molecules were found in close proximity to arginine in bulk solution. This number decreases to around 14 as arginine interacted with OprD binding site and a further reduction was observed when it passed through the narrowest constriction of the pore. Intriguingly, even along the narrowest region there were still as many as 10 water molecules surrounding arginine. Visual inspection reveals that as arginine passed through this region in the vertical orientation described above, some of the water molecules screened the guanidinium group from nearby arginine residues of the basic ladder as its ionic interactions with the negatively charged binding site was broken. The pore was wide enough in this area to allow the passage of these water molecules along with arginine. We note that no ions permeation was observed in any of the windows.

A similar analysis performed on arginine transfer across the bulk outer membrane revealed a strikingly different result. In the LPS headgroup region, as many as 12 water molecules were found around arginine. As arginine breaks interactions between adjacent LPS headgroups, water molecules fill the interstitial void forming hydrogen bonds with either the sugar moieties on LPS or the arginine itself (Figure S7). The deeper arginine travelled into the outer membrane, the more of its hydration 
shell was shed. Around only 5 water molecules remained in the hydrophobic core of the membrane, creating a small energetically unfavourable water-filled cavity. Similar water permeation into the membrane hydrocarbon core was observed during the translocation of ethanol and acetic acid. ${ }^{19}$ Local rearrangement of phospholipids was observed in corresponding windows such that the phosphate headgroups submerged into the centre of the membrane to interact with water molecules as well as the polar groups on arginine as previously described in Figure $5 b$.

a

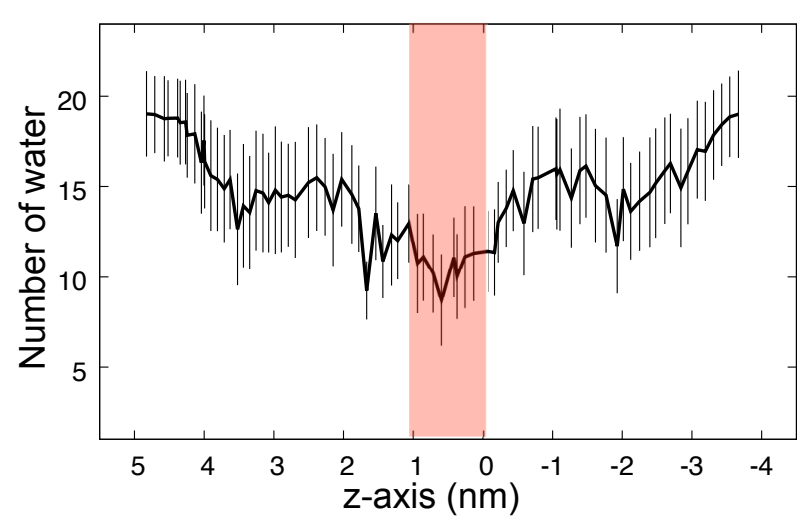

C

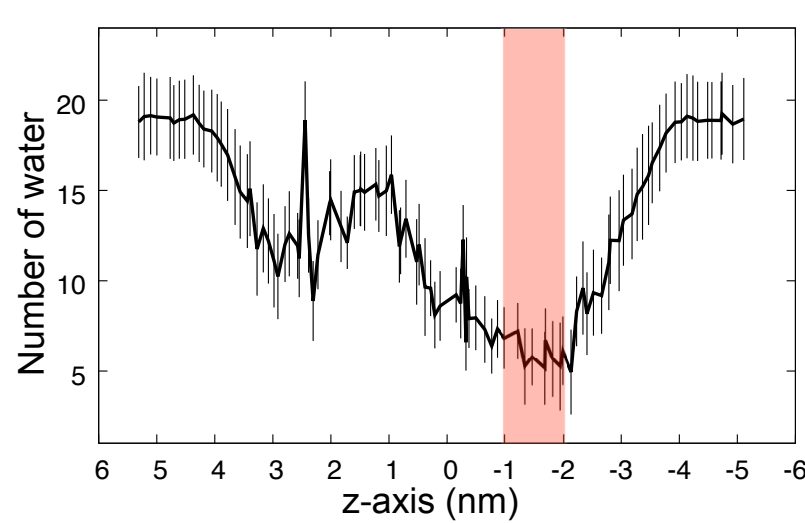

b

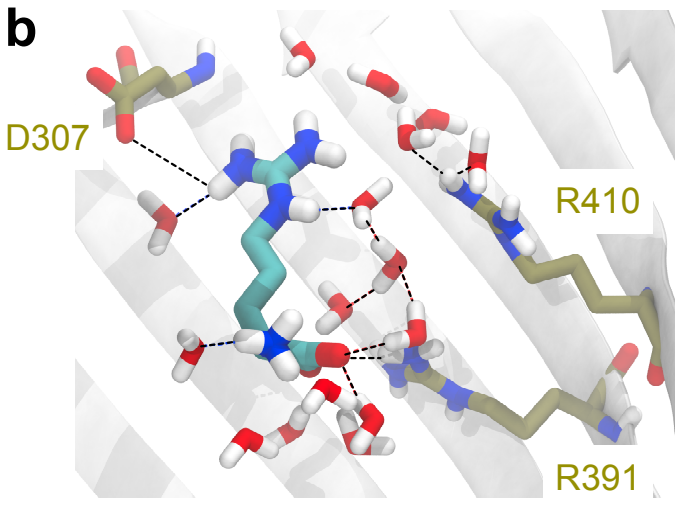

d

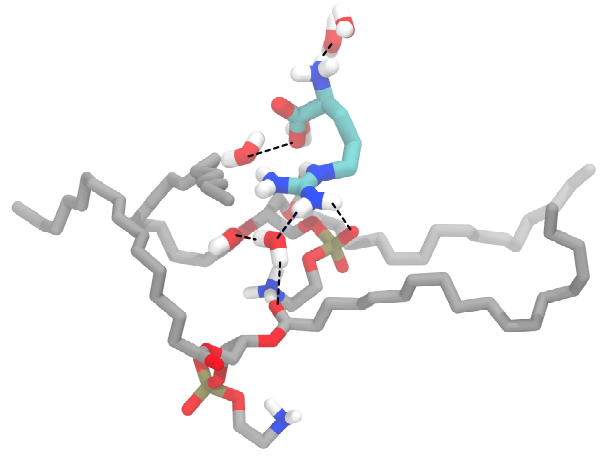

Figure 6. The role of water during arginine permeation. a, The number of water molecules found within $3 \mathrm{~A}$ of arginine during translocation through OprD. Error bars indicate standard deviations. Shaded region indicates the smallest constriction along the pore. $\mathbf{b}, \mathrm{A}$ snapshot from a window within the shaded region highlighting the positions of water molecules with respect to arginine substrate and key residues. Dotted lines show hydrogen bonds and salt bridges. c, The number of water molecules around arginine during permeation through the bulk outer membrane. Shaded region indicates the hydrocarbon tails of the lower leaflet. $\mathbf{d}$, A snapshot from the shaded region illustrating water molecules around arginine with the two closest phospholipids in grey.

\section{Conclusions}

In summary steered MD and umbrella sampling protocols were used to determine the potential of mean force (PMF) of arginine translocation. The PMF along OprD revealed 
a series of energy wells from interactions with LPS headgroups, the putative binding site and the basic ladder nearby the narrowest constriction of the pore. No discernible energy barrier was observed along this pathway, consistent with the fact that the pore remained open throughout our equilibrium simulations, and similar to two other $P$. aeruginosa channels, OprP ${ }^{17,20}$ and OprO ${ }^{21}$, despite our simulations being performed in an asymmetric outer membrane whereas the other two studies were performed in symmetrical phospholipid bilayers. Hydration shells play an important role in screening positively charged moieties of the arginine substrate from the basic ladder of OprD. Interestingly, a nice study by the Jungwirth group ${ }^{51}$ showed guanidinium pairing to stabilise arginine-arginine interactions in aqueous solution and within phospholipid bilayers. We did not observe such interactions between the permeating arginine and the sidechains of the arginine ladder residues, stabilising the interaction was instead with the carboxyl group of the permeating arginine with the guanidinium groups of the ladder residues. This makes sense given that OprD is a conduit for lysine as well as arginine. In bulk outer membrane, arginine was again shown to favourably interact with LPS headgroups, but unlikely to cross the hydrocarbon membrane core due to the large energy barrier. It has been shown in previous simulation studies that the LPS outer core sugars and O-antigens are capable of blocking the entrance of outer membrane porins like $\mathrm{OmpF}^{54}$ and $\mathrm{OpdH},{ }^{55}$ suggesting that small solutes coming from the external environment will have to traverse the thick layer of sugars before contacting any porins and channels. Favourable interactions between LPS and arginine will therefore assist in the initial translocation of the latter prior to OprDmediated transport. Whether the role of this interaction with LPS can be generalised to other solutes is unclear, but certainly worth exploring in future. Taken together, an arginine translocation mechanism across the outer membrane of $P$. aeruginosa thus emerges: i) extracellular arginine molecules partition into the thick layer of LPS headgroups forming hydrogen bonds with the sugar moieties and salt bridges with the phosphate groups, ii) arginine diffuses together with LPS due to the tightly cross-linked LPS headgroups until it encounters an OprD channel, iii) the LPS transfers arginine to the external loops of OprD, iv) arginine spontaneously binds to the putative binding site as it provides the lowest energy minimum, v) the carboxyl group of arginine descended the basic ladder of OprD with water molecules shielding its guanidinium side chain, and vi) at the bottom of the basic ladder, arginine is released into the larger constriction of the channel and eventually into bulk water in the periplasm. Our study 
therefore provides an improved understanding on substrate permeation across a key Pseudomonas porin and may help in future design of antibacterial agents. For example, the guanidinium side chain of arginine has been shown to bind strongly to the putative binding site of $\mathrm{OprD}$, therefore addition of a similar positively charged group to a candidate molecule may improve binding to OprD. Permeation across the channel was facilitated by arginine's ability to maintain an upright position through the most constricted part of the pore. This is due to the interactions between the carboxyl group and the basic ladder, while retaining contact between the guanidium group and the putative binding site. We postulate that having a negatively charged group in a candidate molecule, perhaps $7 \AA$ away from a positively charge moiety as is found in arginine, may improve substrate uptake. Further experimental studies are therefore essential to test the role of incorporating these chemical characteristics towards the uptake of potential antibacterial agents by OprD.

\section{Associated Content}

Supporting Information: Equilibrium simulations of OprD (Figure S1 and S2); snapshots from steered MD simulations of arginine across OprD (Figure S3); sampling and convergence analyses for the umbrella sampling simulations (Figure S4); hydrogen bonding between arginine and LPS and OprD (Figure S5); diffusion of arginine in bulk outer membrane (Figure S6); and snapshots of water molecules surrounding arginine within the LPS headgroup region (Figure S7).

\section{Acknowledgement}

This research was supported by UK Biotechnology and Biological Sciences Research Council (grant BB/M029573/1). This project made use of time on Iridis supercomputers provided by the University of Southampton as well as on ARCHER and JADE provided by HECBioSim.

\section{Reference:}

(1) Nikaido, H. Molecular Basis of Bacterial Outer Membrane Permeability Revisited. Microbiol. Mol. Biol. Rev. 2003, 67 (4), 593-656.

(2) Yoshimura, F.; Nikaido, H. Diffusion of $\beta$-Lactam Antibiotics through the Porin Channels of Escherichia Coli K-12. Antimicrob. Agents Chemother. 1985, 27 (1), 84-92. 
(3) Nestorovich, E. M.; Danelon, C.; Winterhalter, M.; Bezrukov, S. M. Designed to Penetrate: Time-Resolved Interaction of Single Antibiotic Molecules with Bacterial Pores. Proc. Natl. Acad. Sci. U.S.A. 2002, 99 (15), 9789-9794.

(4) Lyczak, J. B.; Cannon, C. L.; Pier, G. B. Establishment of Pseudomonas Aeruginosa Infection: Lessons from a Versatile Opportunist. Microbes Infect. 2000, 2 (9), 1051-1060.

(5) Hancock, R. E. Resistance Mechanisms in Pseudomonas Aeruginosa and Other Nonfermentative Gram-Negative Bacteria. Clin Infect. Dis. 1998, 27, 9399.

(6) Breidenstein, E. B. M.; de la Fuente-Núñez, C.; Hancock, R. E. W. Pseudomonas Aeruginosa: All Roads Lead to Resistance. Trends Microbiol. 2011, 19 (8), 419-426.

(7) Chevalier, S.; Bouffartigues, E.; Bodilis, J.; Maillot, O.; Lesouhaitier, O.; Feuilloley, M. G. J.; Orange, N.; Dufour, A.; Cornelis, P. Structure, Function and Regulation of Pseudomonas Aeruginosa Porins. FEMS Microbiol. Rev. 2017, 41 (5), 698-722.

(8) Eren, E.; Vijayaraghavan, J.; Liu, J.; Cheneke, B. R.; Touw, D. S.; Bryan, W.; Indic, M.; Movileanu, L.; Berg, B. Van Den. Substrate Specificity within a Family of Outer Membrane Carboxylate Channels. PLoS Biol. 2012, 10 (1), e1001242-e1001242.

(9) Trias, J.; Nikaido, H. Outer Membrane Protein D2 Catalyzes Facilitated Diffusion of Carbapenems and Penems Through the Outer Membrane of Pseudomonas Aeruginosa. Antimicrob. Agents Chemother. 1990, 34 (1), 5257.

(10) Shen, J.; Pan, Y.; Fang, Y.; Sekaran, S. D. Role of the Outer Membrane Protein OprD2in Carbapenem-Resistance Mechanisms of Pseudomonas Aeruginosa. PLoS One 2015, 10 (10), 1-9.

(11) Iyer, R.; Sylvester, M. A.; Velez-Vega, C.; Tommasi, R.; Durand-Reville, T. F.; Miller, A. A. Whole-Cell-Based Assay to Evaluate Structure Permeation Relationships for Carbapenem Passage through the Pseudomonas Aeruginosa Porin OprD. ACS Infect. Dis. 2017, 3 (4), 310-319.

(12) Biswas, S.; Mohammad, M. M.; Patel, D. R. Structural Insight into OprD Substrate Specificity. Nat. Struct. Mol. Biol. 2007, 14 (11), 1108-1109.

(13) Eren, E.; Parkin, J.; Adenlawa, A.; Cheneke, B.; Movileanu, L.; Khalid, S.; 
Berg, B. Van Den. Toward Understanding the Outer Membrane Uptake of Small Molecules by Pseudomonas Aeruginosa. J. Biol. Chem. 2013, 288 (17), 12042-12053.

(14) Biswas, S.; Mohammad, M. M.; Movileanu, L.; van den Berg, B. Crystal Structure of the Outer Membrane Protein OpdK from Pseudomonas Aeruginosa. Structure 2008, 16 (7), 1027-1035.

(15) Moraes, T. F.; Bains, M.; Hancock, R. E. W.; Strynadka, N. C. J. An Arginine Ladder in OprP Mediates Phosphate-Specific Transfer across the Outer Membrane. Nat. Struct. Mol. Biol. 2007, 14 (1), 85-87.

(16) Parkin, J.; Khalid, S. Atomistic Molecular-Dynamics Simulations Enable Prediction of the Arginine Permeation Pathway through OccD1/OprD from Pseudomonas Aeruginosa. Biophys. J. 2014, 107 (8), 1853-1861.

(17) Pongprayoon, P.; Beckstein, O.; Wee, C. L.; Sansom, M. S. P. Simulations of Anion Transport through OprP Reveal the Molecular Basis for High Affinity and Selectivity for Phosphate. Proc. Natl. Acad. Sci. U.S.A. 2009, 106, $21614-$ 21618.

(18) Dreyer, J.; Strodel, P.; Ippoliti, E.; Finnerty, J.; Eisenberg, B.; Carloni, P. Ion Permeation in the Nanc Porin from Escherichia Coli: Free Energy Calculations along Pathways Identified by Coarse-Grain Simulations. J. Phys. Chem. B 2013, 117 (43), 13534-13542.

(19) Carpenter, T. S.; Parkin, J.; Khalid, S. The Free Energy of Small Solute Permeation through the Escherichia Coli Outer Membrane Has a Distinctly Asymmetric Profile. J. Phys. Chem. Lett. 2016, 3446-3451.

(20) Modi, N.; Benz, R.; Hancock, R. E. W.; Kleinekathöfer, U. Modeling the Ion Selectivity of the Phosphate Specific Channel Oprp. J. Phys. Chem. Lett. 2012, 3 (23), 3639-3645.

(21) Modi, N.; Ganguly, S.; Barcena-Uribarri, I.; Benz, R.; Berg, B. Van Den; Kleinekatho, U. Structure, Dynamics, and Substrate Specificity of the OprO Porin from Pseudomonas Aeruginosa. Biophys. J. 2015, 109 (October).

(22) DeLano, W. The PyMOL Molecular Graphics System. 2002.

(23) Appelmelk, B. J.; An, Y. Q.; Hekker, T. A. M.; Thijs, L. G.; MacLaren, D. M.; De Graaf, J. Frequencies of Lipopolysaccharide Core Types in Escherichia Coli Strains from Bacteraemic Patients. Microbiology 1994, 140 (5), 1119-1124.

(24) Vinogradov, E. V.; Van Der Drift, K.; Thomas-Oates, J. E.; Meshkov, S.; Brade, 
$\mathrm{H}$.; Holst, O. The Structures of the Carbohydrate Backbones of the Lipopolysaccharides from Escherichia Coli Rough Mutants F470 (R1 Core Type) and F576 (R2 Core Type). Eur. J. Biochem. 1999, 261 (3), 629-639.

(25) Lugtenberg, E. J. .; Peters, R. Distribution of Lipids in Cytoplasmic and Outer Membranes of Escherichia Coli K12. Biochim. Biophys. Acta 1976, 441 (195 6), 38-47.

(26) Aibara, S.; Kato, M.; Ishinaga, M.; Kito, M. Changes in Positional Distribution of Fatty Acids in the Phospholipids of Escherichia Coli after Shift-Down in Temperature. Biochim. Biophys. Acta 1972, 270, 301-306.

(27) Kito, M.; Ishinaga, M.; Nishihara, M.; Kato, M.; Sawada, S. Metabolism of the Phosphatidylglycerol Molecular Species in Escherichia Coli. Eur. J. Biochem. 1975, 54 (1), 55-63.

(28) Yokota, K.; Kanamoto, R.; Kito, M. Composition of Cardiolipin Molecular Species in Escherichia Coli. J. Bacteriol. 1980, 141 (3), 1047-1051.

(29) Piggot, T. J.; Holdbrook, D. A.; Khalid, S. Electroporation of the E. Coli and S. Aureus Membranes: Molecular Dynamics Simulations of Complex Bacterial Membranes-Suppinfo. J. Phys. Chem. 2011.

(30) Ortiz-Suarez, M. L.; Samsudin, F.; Piggot, T. J.; Bond, P. J.; Khalid, S. FullLength OmpA: Structure, Function, and Membrane Interactions Predicted by Molecular Dynamics Simulations. Biophys. J. 2016, 111 (8), 1692-1702.

(31) Samsudin, F.; Boags, A.; Piggot, T. J.; Khalid, S. Braun's Lipoprotein Facilitates OmpA Interaction with the Escherichia Coli Cell Wall. Biophys. J. 2017, 113 (7), 1496-1504.

(32) Saunders, G. M.; Bruce Macdonald, H. E.; Essex, J. W.; Khalid, S. Prediction of the Closed Conformation and Insights into the Mechanism of the Membrane Enzyme LpxR. Biophys. J. 2018, 115 (8), 1445-1456.

(33) Wolf, M. G.; Hoefling, M.; Aponte-santamaría, C.; Grubmüller, H.; Groenhof, G. G_membed : Efficient Insertion of a Membrane Protein into an Equilibrated Lipid Bilayer with Minimal Perturbation. J. Comp. Chem. 2010, 31 (11), 21692174.

(34) Bussi, G.; Donadio, D.; Parrinello, M. Canonical Sampling through Velocity Rescaling. J. Chem. Phys. 2007, 126 (1), 014101.

(35) Parrinello, M.; Rahman, A. Polymorphic Transitions in Single Crystals: A New Molecular Dynamics Method. J. Appl. Phys. 1981, 52 (12), 7182-7190. 
(36) Hess, B.; Bekker, H.; Berendsen, H. J. C.; Fraaije, J. G. E. M. LINCS: A Linear Constraint Solver for Molecular Simulations. J. Comp. Chem. 1997, 18 (12), 1463-1472.

(37) Essmann, U.; Perera, L.; Berkowitz, M. L.; Darden, T.; Lee, H.; Pedersen, L. G. A Smooth Particle Mesh Ewald Method. J. Chem. Phys. 1995, 103 (19), 8577-8593.

(38) Abraham, M. J.; Murtola, T.; Schulz, R.; Páall, S.; Smith, J. C.; Hess, B.; Lindah, E. Gromacs: High Performance Molecular Simulations through MultiLevel Parallelism from Laptops to Supercomputers. SoftwareX 2015, 1-2, 1925.

(39) Oostenbrink, C.; Villa, A.; Mark, A. E.; van Gunsteren, W. F. A Biomolecular Force Field Based on the Free Enthalpy of Hydration and Solvation: The GROMOS Force-Field Parameter Sets 53A5 and 53A6. J. Comput. Chem. 2004, 25 (13), 1656-1676.

(40) Berendsen, H. J.; Postma, J. P. M.; van Gunsteren, W. F.; Hermans, J. Interaction Models for Water in Relation to Protein Hydration. In Intermolecular Forces; Pullman, B., Ed.; Reidel Publishing: Boston, 1981; pp 331-342.

(41) Humphrey, W.; Dalke, A. VMD: Visual Molecular Dynamics. J. Mol. Graph. 1996, 15, 33-38.

(42) Kumar, S.; Rosenberg, J. M.; Bouzida, D.; Swendsen, R. H.; Kollman, P. A. THE Weighted Histogram Analysis Method for Free-Energy Calculations on Biomolecules. I. The Method. J. Comput. Chem. 1992, 13 (8), 1011-1021.

(43) Hub, J. S.; De Groot, B. L.; Van Der Spoel, D. G-Whams-a Free Weighted Histogram Analysis Implementation Including Robust Error and Autocorrelation Estimates. J. Chem. Theory Comput. 2010, 6 (12), 3713-3720.

(44) Somboon, K.; Niramitranon, J.; Pongprayoon, P. Probing the Binding Affinities of Imipenem and Ertapenem for Outer Membrane Carboxylate Channel D1 (OccD1) from P. Aeruginosa: Simulation Studies. J. Mol. Model. 2017, 23 (8).

(45) Isabella, V. M.; Campbell, A. J.; Manchester, J.; Sylvester, M.; Nayar, A. S.; Ferguson, K. E.; Tommasi, R.; Miller, A. A. Toward the Rational Design of Carbapenem Uptake in Pseudomonas Aeruginosa. Chem. Biol. 2015, 22 (4), 535-547.

(46) Samanta, S.; Scorciapino, M. A.; Ceccarelli, M. Molecular Basis of Substrate Translocation through Outer Membrane Channel OprD of Pseudomonas 
Aeruginosa. Phys. Chem. Chem. Phys. 2015, 17, 23867-23876.

(47) Arunmanee, W.; Pathania, M.; Solovyova, A. S.; Le Brun, A. P.; Ridley, H.; Baslé, A.; van den Berg, B.; Lakey, J. H. Gram-Negative Trimeric Porins Have Specific LPS Binding Sites That Are Essential for Porin Biogenesis. Proc. Natl. Acad. Sci. U.S.A. 2016, 201602382.

(48) Chorev, D. S.; Baker, L. A.; Wu, D.; Beilsten-Edmands, V.; Rouse, S. L.; ZeevBen-Mordehai, T.; Jiko, C.; Samsudin, F.; Gerle, C.; Khalid, S.; et al. Protein Assemblies Ejected Directly from Native Membranes Yield Complexes for Mass Spectrometry. Science (80-. ). 2018, 362 (6416), 829-834.

(49) Sehnal, D.; Vařeková, R. S.; Berka, K.; Pravda, L.; Navrátilová, V.; Banáš, P.; Ionescu, C. M.; Otyepka, M.; Koča, J. MOLE 2.0: Advanced Approach for Analysis of Biomacromolecular Channels. J. Cheminf. 2013, 5 (8), 1-13.

(50) Fitch, C. A.; Platzer, G.; Okon, M.; Garcia-Moreno, B. E.; Mclntosh, L. P. Arginine: Its PKavalue Revisited. Protein Sci. 2015, 24 (5), 752-761.

(51) Allolio, C.; Baxova, K.; Vazdar, M.; Jungwirth, P. Guanidinium Pairing Facilitates Membrane Translocation. J. Phys. Chem. B 2016, 120 (1), 143153.

(52) Reif, M. M.; Winger, M.; Oostenbrink, C. Testing of the GROMOS Force-Field Parameter Set 54A8: Structural Properties of Electrolyte Solutions, Lipid Bilayers, and Proteins. J. Chem. Theory Comput. 2013, 9 (2), 1247-1264.

(53) Hsu, P. C.; Samsudin, F.; Shearer, J.; Khalid, S. It's Complicated: Curvature, Diffusion and Lipid Sorting Within the Two Membranes of Escherichia Coli. J. Phys. Chem. Lett. 2017, 8, 5513-5518.

(54) Patel, D. S.; Re, S.; Wu, E. L.; Qi, Y.; Klebba, P. E.; Widmalm, G.; Yeom, M. S.; Sugita, Y.; Im, W. Dynamics and Interactions of OmpF and LPS: Influence on Pore Accessibility and Ion Permeability. Biophys. J. 2016, 110 (4), 930938.

(55) Lee, J.; Pothula, K. R.; Kleinekathöfer, U.; Im, W. Simulation Study of Occk5 Functional Properties in Pseudomonas Aeruginosa Outer Membranes. J. Phys. Chem. B 2018, 122 (34), 8185-8192. 
TOC Graphic

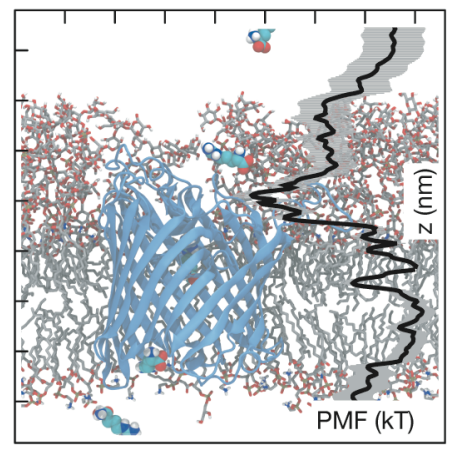

